INPLASY

PROTOCOL

To cite: Chun et al. Role of acupuncture in the treatment of COPD: an overview of systematic reviews and metaanalyses. Inplasy protocol 2020110090. doi:

10.37766/inplasy2020.11.0090

Received: 20 November 2020

Published: 21 November 2020

Corresponding author: Jiansheng $\mathbf{L i}$

li_js8@163.com

Author Affiliation:

Longhua Hospital Shanghai University of Traditional Chinese Medicine

Support: Key program of NNSF.

Review Stage at time of this submission: Preliminary searches.

Conflicts of interest: There are no conflicts of interest.

\section{Role of acupuncture in the treatment of COPD: an overview of systematic} reviews and meta-analyses

Chun, L1; Li, XL2; Feng, ZZ3; Xie, Y4; Li, JS55.

Review question / Objective: What are the effects of acupuncture for chronic obstructive pulmonary disease (COPD)? A certain number of systematic reviews have been published to provide corresponding evidence support for it. However, through preliminary research, it is found that the results of different system evaluations are not completely consistent, the quality of research is not the same, and the level of clinical evidence cannot be determined, there is no consistent evidence on the effectiveness of acupuncture in the treatment of COPD. In this study, AMSTAR 2 list and GRADE system will select to comprehensively evaluate the methodology and evidence quality level of acupuncture treatment of COPD, and to evaluate its applicability and safety, so as to provide reference for future research on highquality acupuncture treatment of COPD.

INPLASY registration number: This protocol was registered with the International Platform of Registered Systematic Review and Meta-Analysis Protocols (INPLASY) on 21 November 2020 and was last updated on 21 November 2020 (registration number INPLASY2020110090).

\section{INTRODUCTION}

Review question / Objective: What are the effects of acupuncture for chronic obstructive pulmonary disease (COPD)? A certain number of systematic reviews have been published to provide corresponding evidence support for it. However, through preliminary research, it is found that the results of different system evaluations are not completely consistent, the quality of research is not the same, and the level of clinical evidence cannot be determined, there is no consistent evidence on the effectiveness of acupuncture in the treatment of COPD. In this study, AMSTAR 
2 list and GRADE system will select to comprehensively evaluate the methodology and evidence quality level of acupuncture treatment of COPD, and to evaluate its applicability and safety, so as to provide reference for future research on highquality acupuncture treatment of COPD.

Condition being studied: Acupuncture, as a traditional treatment, has a long history of use for a range of health conditions, including respiratory diseases, and numerous randomised, controlled trials (RTCs) have evaluated the clinical efficacy of acupuncture. And it has suggested that adverse events are rare and often minor. In the newest GOLD report, acupuncture could alleviat dyspnea and improved the quality of life of patients with advanced COPD. In recent years, with the increasing number of clinical studies on acupuncture treatment of COPD, a certain number of systematic reviews have been published to provide corresponding evidence support for clinical decision-making.

\section{METHODS}

Search strategy: An electronic literature search was conducted in the PubMed, Embase, Cochrane Library, Web of Science, CNKI, CBM, VIP and WanFang database, all from the inception to September 2020. There were no restrictions on the source, publication date, or language of the SRs/ MAs. The search terms and basic search strategy were as follows: ("COPD" OR "Chronic Obstructive Pulmonary Disease" OR "COAD" OR "Chronic Obstructive Lung Disease" OR "Pulmonary Disease, Chronic Obstructive") AND ("acupuncture" OR "manual acupuncture" OR "electroacupuncture" OR "auricular acupuncture" OR "warm acupuncture" OR "acupoints") AND ("systematic review" OR "meta-analysis" OR "systematic assessment"). In addition, to ensure a comprehensive data collection, references of relevant reviews were searched manually to identify additional eligible studies.
Participant or population: Patients with COPD and were diagnosed according to any internationally recognized clinical guidelines.

Intervention: Treatment measures in the intervention group included various acupuncture therapies (e.g. acupuncture, electroacupuncture, auricular acupuncture, and body acupuncture) or acupuncture combined with other therapies, yet noninvasive techniques, such as single moxibustion, acupressure, point application, laser acupuncture were excluded.

Comparator: Treatments in the control group included comfort therapy (e.g. a placebo, sham acupuncture, or a blank control) or other therapies (e.g., medication therapy or other nondrug therapies).

Study designs to be included: Systematic reviews/meta-analyses of randomized controlled trials.

Eligibility criteria: We included studies which met the following criteria: 1)Study Design and Population: SRs/Mas in which the participants were patients with COPD and were diagnosed according to any internationally recognized clinical guidelines; 2)Interventions: Treatment measures in the intervention group included various acupuncture therapies (e.g. acupuncture, electroacupuncture, auricular acupuncture, and body acupuncture) or acupuncture combined with other therapies, yet noninvasive techniques, such as single moxibustion, acupressure, point application, laser acupuncture were excluded. Treatments in the control group included comfort therapy (e.g. a placebo, sham acupuncture, or a blank control) or other therapies (e.g., medication therapy or other nondrug therapies); 3)Outcomes: reporting on at least one of the pre-specified outcomes: (1) lung function (FEV1\%pre, FEV1/FVC), (2) health related quality of life (e.g. St. George's Respiratory Questionnaire (SGRQ)), (3) exercise capacity (e.g. distance walked in six minutes $(6 M W T))$, No 
restriction was placed on gender or stage of COPD (e.g. stable or acute exacerbation).

Information sources: We review the references of included literatures to avoid the occurrence omission. Also, we will contact with author in case that we could not obtain full text.

Main outcome(s): Reporting on at least one of the pre-specified outcomes: (1) lung function (FEV1\%pre, FEV1/FVC), (2) health related quality of life (e.g. St. George's Respiratory Questionnaire (SGRQ)), (3) exercise capacity (e.g. distance walked in six minutes (6MWT)), No restriction was placed on gender or stage of COPD (e.g. stable or acute exacerbation).

Quality assessment / Risk of bias analysis: The methodological quality of included SRs/MAs will be assessed by the Assessment of Multiple Systematic Reviews 2 (AMSTAR 2). The quality of evidence for outcomes of FEV1\%pre, FEV1/ FVC, 6MWD and SGRQ will be assessed using the Grading of Recommendations, Assessment, Development and Evaluation (GRADE).

Strategy of data synthesis: We will report the results narratively in text and tables, together with comments on the certainty of the evidence. We organized the data by intervention target and second by type of reported main outcomes.

Subgroup analysis: Subgroup analysis will be conducted based on the different interventions:(日) acupuncture plus medicine vs medicine;(月) acupuncture vs sham acupuncture; (火)acupuncture plus pulmonary rehabilitation vs pulmonary rehabilitation.

Sensibility analysis: Not applicable.

Language: There is no language limits on the search.

Country(ies) involved: China.
Keywords: acupuncture; chronic obstructive pulmonary disease; overview; AMSTAR-2; GRADE.

Contributions of each author:

Author 1 - Liu Chun.

Author 2 - Xuanlin Li.

Author 3 - Zhenzhen Feng.

Author 4 - Yang Xie.

Author 5 - Jiansheng Li. 